\title{
ESTILOS DE ENSEÑANZA E INVOLUCRAMIENTO EN EL TRABAJO EN DOCENTES DE UNA UNIVERSIDAD PRIVADA DE AREQUIPA (PERÚ)
}

\author{
Teaching styles and job involvement in lecturers from \\ a private university at Arequipa (Peru)
}

\author{
Walter L. Arias Gallegos* y Andrea B. Carrasco Orozco** \\ Universidad Católica San Pablo, Arequipa, Perú
}

\section{Resumen}

En este artículo se valoran las relaciones entre los estilos de enseñanza y el involucramiento laboral de profesores de una universidad privada de la ciudad de Arequipa. Para ello, se evaluó a 120 profesores universitarios mediante el Cuestionario de Estilos de Enseñanza de Grasha y la Escala de Involucramiento en el Trabajo de Lodahl y Kejner. Los datos fueron comparados en función del sexo, el grado de instrucción y la profesión de los docentes, se realizaron correlaciones y análisis de regresión para valorar la relación entre las variables de estudio. Los resultados indican que los estilos de enseñanza más predominantes entre los profesores de la muestra fueron el Experto, el Facilitador y de Modelo personal. Asimismo, las dimensiones más altas del involucramiento laboral fueron el Compromiso con el trabajo y el Fluir en el trabajo. El involucramiento en el trabajo se relacionó de manera moderada con los estilos Experto y Modelo personal, pero solo el estilo de enseñanza Experto tuvo impacto positivo y significativo en el involucramiento en el trabajo.

Palabras clave: Estilos de enseñanza, involucramiento en el trabajo, docente universitario.

\begin{abstract}
In this article, the relationships between teaching styles and job involvement in lecturers, from a private university of Arequipa City, are valued. For that purpose, 120 university lecturers were tested with Grasha's Teaching Styles Questionnaire and Lodahl and Kejner's Job Involvement Scale. The data was compared according to sex, educational level and the profession of the lecturers, correlations and a regression analysis were made in order to value the relationship between the variables of the study. The results indicate that the most predominant teaching styles between the lecturers of the sample were: Expert, Facilitator and Personal model. Moreover, the highest job involvement dimensions were job commitment and job flow. The job involvement was moderately related to the Expert and Personal model teaching styles, but only the Expert style had a positive and significant impact in job involvement.
\end{abstract}

Key words: Teaching styles, job involvement, university lecturer.

\footnotetext{
* Psicólogo. Universidad Católica San Pablo.Arequipa, Perú. warias@ucsp.edu.pe

** Marketing. Universidad Católica San Pablo. Arequipa,
} 


\section{INTRODUCCIÓN}

Desde hace varias décadas, se ha puesto especial énfasis en los estilos de aprendizaje de los estudiantes, como un enfoque personalizado de enseñanza, que se basa en las cualidades cognitivas de los aprendices; de manera tal que se puedan diseñar estrategias más pertinentes según sus preferencias individuales en un contexto académico. Los estilos de aprendizaje han sido objeto de estudio, en diversos escenarios educativos y niveles de enseñanza, pero suele haber un predominio de estudios en estudiantes de nivel superior. Esto podría deberse a que es en este nivel, que los estudiantes asumen de manera más consciente su propio aprendizaje.

En Arequipa, los estilos de aprendizaje han sido objeto de estudio de Portilla y Barreda (2002), quienes evaluaron los estilos de aprendizaje en estudiantes de artes e ingenierías de la Universidad Nacional de San Agustín; así como los trabajos de Arias, que evaluó los estilos de aprendizaje de estudiantes universitarios de cuatro carreras profesionales, y encontró que en los primeros años predomina un estilo de aprendizaje divergente, pero en los últimos semestres de estudio, el estilo asimilador era el más frecuente (Arias, 2011). También reportó que el estilo de aprendizaje asimilador se asocia con una mayor capacidad intelectual (Arias, 2014) y que los estilos de aprendizaje no tienen efecto predictivo sobre las estrategias metacognitivas de estudiantes de psicología de tres universidades de Arequipa (Arias, Zegarra, \& Justo, 2014).

Por otro lado, más recientemente, se ha insertado en el campo de la educación, el concepto de estilos de enseñanza, que hace alusión a la "forma peculiar que tiene cada profesor de elaborar el programa, aplicar el método, organizar la clase y relacionarse con los alumnos" (Ferrández y Sarramona, 1987, citados por Martínez, 2009). Otras definiciones señalan que se trata de las metodologías y estrategias que los profesores utilizan durante la enseñanza (Khurshid, \& Aurangzeb, 2012).

$\mathrm{Al}$ respecto, un autor que ha desarrollado un modelo teórico sobre los estilos de enseñanza es Anthony Grasha. Este autor, comenzó investigando los estilos de aprendizaje, para luego centrarse en los estilos de enseñanza (Grasha, 1995). Según su modelo se pueden distinguir cinco estilos de enseñanza: estilo experto, autoridad formal, modelo personal, facilitador y delegador, que pueden estar influidos por aspectos tales como la naturaleza del curso, el nivel de aprendizaje de los estudiantes, el tiempo para el dictado de clases y otras cuestiones contextuales de las instituciones educativas (Grasha, 2000).

En ese sentido, se ha visto que los estilos de enseñanza, también están influidos por la concepción de aprendizaje que tienen los profesores (Sánchez, 2005), su estilo cognitivo (Meneses, 2013), sus estilos de aprendizaje (Polanco-Bueno, 1999), su personalidad (Pastor, 2017) y el tipo de clase (teóricas vs. práctica) y la disciplina que se imparte (ciencias exactas vs. humanidades) (Borgobello, Peralta, \& Roselli, 2010). También se ha visto que, cuando los estudiantes brindan retroalimentación al docente, este puede cambiar sus estilos de enseñanza (PolancoBueno, 1994), y que el proceso de evaluación docente afecta su desenvolvimiento en clases (Denazis, \& Óscar, 2000).

De acuerdo con el modelo teórico de Grasha (2000), los docentes con un estilo de enseñanza tipo Experto, basan su estilo de enseñanza en el dominio del tema que poseen y promueven la competencia entre los estudiantes. El estilo de enseñanza tipo Autoridad formal, basa su enseñanza en el estatus del profesor, y aunque también relieva el dominio del tema, se centra más en el cumplimiento de las normas institucionales dentro del proceso de enseñanza-aprendizaje. Estos profesores son buenos dando retroalimentación a sus estudiantes El estilo de enseñanza tipo Modelo personal se caracteriza porque enseña a sus estudiantes a través del ejemplo, tanto de su forma de pensar como de comportarse. Este estilo enfatiza la educación moral de los educandos. El estilo de enseñanza tipo Facilitador, promueve la independencia gradual de los estudiantes y se basa en el desarrollo del pensamiento crítico a través de técnicas de pedagogía activa. El estilo de enseñanza tipo Delegador, tiene un carácter más consultivo, pues proporciona autonomía al estudiante y se basa en su capacidad de autodirección.

En cada caso, los estilos de enseñanza, promueven un tipo de relación con el estudiante en función de las características del profesor. Esta relación tiene un 
carácter dinámico y es recíproca, de modo tal que, de la interacción entre el profesor y el estudiante, surge un modelo de enseñanza-aprendizaje que tiene repercusiones positivas para ambos (Grasha, 1985). Por eso, así como es importante, conocer y evaluar los estilos de aprendizaje del estudiante, también es importante conocer y valorar los estilos de enseñanza del profesor (Grasha, 1995).

Ahora bien, los cinco estilos de enseñanza de Grasha conforman cuatro clusters que surgen de la combinación de las características afines entre los estilos de enseñanza (Grasha, 1995). De este modo, el primer cluster combina de manera primaria los estilos Experto y de Autoridad formal; el segundo cluster combina los estilos Experto, Modelo personal y de Autoridad formal; el tercer cluster comprende los estilos Experto, Facilitador y Modelo personal; y el cuarto cluster combina los estilos Experto, Facilitador y Delegador (Grasha, 2000).

Estas combinaciones ofrecen una visión más concreta de los estilos de enseñanza, pues difícilmente, un profesor posee un estilo de enseñanza puro. En ese sentido, diversos estudios han explorado el alcance del modelo de Grasha. Por ejemplo, se ha visto que los estilosdeenseñanza promueveneldesarrollopsicosocial de los estudiantes (Khurshid, \& Aurangzeb, 2012) y favorecen notablemente su compromiso académico (Shaari, Ysoff, Ghazali, Osman, \& Dzahir, 2014). Además, se ha reportado que cuando los profesores hacen uso adecuado de sus estilos de enseñanza, los estudiantes se comunican mejor (Aguilera, 2014).

Por otro lado, se ha visto que muchas veces los estilos de enseñanza de los profesores según el modelo de Grasha no concuerdan con los estilos de aprendizaje de los estudiantes (Kassaian, \& Ayatollahi, 2010), Otros estudios, tampoco han encontrado relación entre los estilos de aprendizaje del profesor y el estilo de aprendizaje del alumno (Segura, 2011). Sin embargo, es importante considerar el modelo teórico del cual se parte para conceptualizar y diferenciar los diversos tipos de estilos de aprendizaje, así como los estilos de enseñanza.

Así, por ejemplo, el modelo teórico de Alonso, Gallego y Honey, propone que un estilo de enseñanza abierto es compatible con un estilo de aprendizaje activo, un estilo de enseñanza formal es compatible con un estilo de aprendizaje reflexivo, un estilo de enseñanza estructurado es compatible con un estilo de aprendizaje teórico, y un estilo de enseñanza funcional es compatible con un estilo de aprendizaje pragmático (Martínez,2009).En otros casos, se ha podido confirmar que los estilos de aprendizaje de Kolb se relacionan con los estilos de enseñanza de Grasha. Por ejemplo, en el estudio de Rojas, Zárate y Lozano (2016) el estilo Experto/Autoridad formal se relacionó con el estilo de aprendizaje asimilador en el $83 \%$ de los casos; sin embargo, los autores concluyen que "la apropiación de los conocimientos desde los diferentes estilos de aprendizaje no depende exclusivamente de la relación que se teja entre los estilos de enseñanza del profesor y los estilos de aprendizaje de los estudiantes, sino sobre todo del desarrollo de metodologías variadas" (p. 201).

En ese sentido, es indudable que la labor docente tiene un impacto favorable o no, sobre los estudiantes, y no solo a nivel académico, sino también personal (Aguilera, \& Ortiz, 2011). De ahí, la necesidad de valorar los estilos de enseñanza de los profesores, sin embargo, en el Perú casi no existen estudios sobre dicha variable, salvo una investigación sobre estilos de aprendizaje en profesores universitarios, que se realizó en 459 docentes de la Pontificia Universidad Católica del Perú, en el que se reportó que el estilo predominante era el reflexivo, según el modelo de Honey y Alonso, y que no habían diferencias significativas en función de las variables demográficas y laborales de los profesores (Coloma, Manrique, Revilla, \& Tafur, 2008).

En la ciudad de Arequipa, Pastor (2017) evaluó los estilos de enseñanza de 50 docentes de un instituto de nivel superior, con la finalidad de valorar la relación con su personalidad, y reportó que los profesores más extrovertidos tendían a tener un estilo de enseñanza de tipo Delegador. Más allá de estos dos trabajos de investigación, no hay estudios sobre los estilos de enseñanza en relación con aspectos laborales como el involucramiento en el trabajo. Por esta variable se entiende el grado en que un individuo se orienta o está determinado por su trabajo (Martínez-Lugo, 1988), así como la identificación que tiene con su trabajo y la absorción que siente cuando está realizando sus 
funciones laborales (Lawler, \& Hall, 1970). De ahí que el involucramiento en el trabajo posee componentes cognitivos, afectivos y conductuales (Nazem, \& Mozaiini, 2014).

A nivel laboral, los profesores peruanos están sometidos a unagran diversidad de riesgos psicosociales que afectan su desenvolvimiento docente, por ello sería importante valorar el grado de relación entre los estilos de enseñanza y su involucramiento en el trabajo, pues estudios previos, han reportado que los profesores suelen tener altos índices de síndrome de burnout, de manera que los profesores que laboran en instituciones educativas públicas tienen altos niveles de despersonalización mientras que los profesores de instituciones educativas privadas tienen altos niveles de baja realización profesional (Arias, \& Jiménez, 2013).

En ese sentido, el síndrome de burnout suele oponerse conceptual y teóricamente con el engagement, que hace referencia al grado de compromiso que tiene la persona con su trabajo. Aunque el involucramiento y el compromiso en el trabajo se encuentran íntimamente relacionados, no son lo mismo (Khan, Jam, Akbar, Khan, \& Hijazi, 2011), ya que el primero se orienta más hacia la organización y el segundo hacia el trabajo propiamente dicho (Ekmekçi, 2011). Asimismo, mientras el síndrome de burnout se caracteriza por el agotamiento emocional, la despersonalización y la baja realización personal, el engagement, se compone de la energía, el involucramiento en el trabajo y la absorción (Castellano, Cifré, Spontón, Medrano, \& Maffei, 2013). Es decir, mientras el burnout se refiere a un conjunto de síntomas negativos que perjudican el desempeño laboral del trabajador, el engagement, hace alusión a características positivas del trabajador que promueven su productividad laboral.

De hecho, el involucramiento en el trabajo se ha relacionado positivamente con la satisfacción laboral (Saedi, \& Iravani, 2016), la motivación intrínseca (Lowler, \& Hall, 1970), el compromiso en el trabajo (Blau, \& Boal, 1987), el apoyo social (Mishra, \& Shyam, 2005), el liderazgo transformacional (Nazem, \& Mozaiini, 2014), la cultura organizacional (Rizvi, 2013), la autoeficacia (Hui-Ling, Yu-Hsiu, \& Yi-Ching, 2006), la implicación emocional (Lucia-Casademunt,
Morales-Gutiérrez, \& Ariza-Montes, 2012), el locus de control interno, (Van Wyk, Boshoff, \& Cilliers, 2003), el civismo organizacional (Saxena, \& Saxena, 2015), y ciertos rasgos de personalidad, como la extroversión, la cordialidad y la responsabilidad (Chi-Shun, \& ChengWen, 2009). Mientras que, por el contrario, mantiene relaciones negativas con el estrés marital (Uzonwanne, 2014), la inestabilidad emocional (Mahajan, 2015) y el estrés laboral (López-Araujo, Osca, \& Peiró, 2007).

Porotrolado,algunascondicionesorganizacionales suelen favorecer el involucramiento en el trabajo, como el clima organizacional (Biswas, 2009), la autonomía en el trabajo (Gilkar, \& Darzi, 2012), la estructura organizacional (Deosthalee, 2004), y la naturaleza del trabajo. Por ejemplo, se ha reportado que las profesoras de nivel preescolar, se sienten emocionalmente reforzadas por el contacto que tienen con niños pequeños y la responsabilidad que ello implica, lo que incrementa su involucramiento en el trabajo (Ching-Sheue, 2015). De hecho, las personas que tienen elevados niveles de involucramiento en el trabajo, consideran sus obligaciones laborales como aspectos esenciales de sus vidas (Nwibere, 2014).

Ahora bien, a pesar de que el concepto de involucramiento en el trabajo fue introducido por Lodahl y Kejner en la década de los 60 (Lodahl, \& Kejner, 1965), fue a finales de los 80 que se iniciaron los estudios sobre este constructo en América Latina, gracias a las investigaciones de Martínez-Lugo (1988, 1990; Andújar y Martínez-Lugo, 1996). Mientras que, en el Perú, no se han reportado estudios sobre esta variable, a excepción de una investigación psicométrica con 223 trabajadores de la ciudad de Arequipa (Arias, \& Jiménez, 2017).En tal sentido, la presente investigación pretende responder a las siguientes preguntas de investigación: ¿cómo se manifiesta el involucramiento en el trabajo y los estilos de enseñanza en profesores de una universidad privada de Arequipa, en función del sexo, el grado académico y la profesión?, ¿cuál es el grado de relación entre los estilos de enseñanza y el involucramiento en el trabajo?, y ¿qué efecto tiene el involucramiento laboral en los estilos de enseñanza y viceversa? 


\section{MÉTODO}

Se planteó una investigación de tipo exploratoria, pues no existen datos sobre las variables que son objeto de estudio (Hernández, Fernández, \& Baptista, 2011) y se trabajó con un diseño de investigación de tipo asociativo-predictivo (Ato, López, \& Benavente, 2013).

\section{Participantes}

La muestra está compuesta por 120 profesores universitarios, que corresponden al 20\% de una población de 600 profesores de una universidad privada de la ciudad de Arequipa. El 60\% son varones y el $40 \%$ son mujeres. La edad promedio es de 39 años con una desviación típica de \pm 11.27 , entre un rango de 22 y 82 años. El $77.5 \%$ son titulados, el $17.5 \%$ tienen el grado de Maestro y el 5\% el grado de Doctor. En cuanto a su profesión, el $7.5 \%$ son administradores, el $3.33 \%$ economistas, el $1.66 \%$ periodistas, el $6.66 \%$ son psicólogos, el $24.16 \%$ son ingenieros, el $32.5 \%$ son educadores, el $12.5 \%$ son abogados, el $3.33 \%$ son biólogos, el $0.83 \%$ son contadores y el $7.5 \%$ son especialistas en otras áreas. El método de selección fue no probabilístico mediante la técnica de muestreo por cuotas (Hernández, Fernández, \& Baptista, 2011).

\section{Instrumentos}

Se ha utilizado el Cuestionario de Estilos de Enseñanza de Grasha (1996), que consta de 40 reactivos con siete alternativas de respuesta en una escala tipo Likert que van desde Totalmente de acuerdo hasta Totalmente en desacuerdo. Cuenta con cinco factores que representan los estilos de enseñanza propuestos por Grasha: Experto, Autoridad formal, Modelo personal, Facilitador y Delegador. Cada factor se compone de ocho ítems cuya sumatoria, ofrece puntuaciones que representan niveles bajos, medios o altos de la presencia de características propias de los estilos de enseñanza. La prueba ha sido validada por Rojas, Zárate y Lozano (2016), para muestras latinoamericanas, con buenos indicadores psicométricos.

También se utilizó la Escala de Involucramiento en el Trabajo de Lodahl y Kejner que consta de 20 reactivos con cinco alternativas de respuesta en una escala tipo Likert que va desde muy de acuerdo (5) hasta muy desacuerdo (1). La escala tiene una estructura de siete factores (Andújar y Martínez-Lugo, 1996): Factor negativo (ítems 10, 13, 18 y 19), Preocupación por el trabajo (ítems 7, 9 y 2), Proactividad (ítems 5, 6 y 12), Sentido del trabajo (ítems 1,2 y 3), Importancia del trabajo (ítems 11 y 14), Compromiso (ítems 16 y 17) y Fluir en el trabajo (ítems 4, 8 y 15). Este instrumento cuenta con validez y confiabilidad, reportados por Andújar y Martínez-Lugo (1996) para muestras latinoamericanas, y por Arias y Jiménez (2017) para muestras de la ciudad de Arequipa.

\section{Procedimientos}

En primer término, se procedió a solicitar los permisos correspondientes ante las autoridades universitarias. Una vez obtenidos, se procedió a evaluar a los docentes en la sala de profesores, siempre que quisieron participar voluntariamente en el estudio. Se les explicó los fines de la investigación y se aplicaron los instrumentos en un tiempo aproximado de 20 minutos por profesor. El recojo de la información se hizo a finales del año 2016, entre los meses de noviembre y diciembre. Los datos fueron codificados y procesados mediante el programa SPSS versión 21, según el nivel de medición de las variables y las interrogantes de investigación. De este modo, se realizaron análisis descriptivos de las medidas de tendencia central y las medidas de dispersión, comparaciones con la prueba $t$ de Student y el análisis de varianza, correlaciones con el coeficiente de Pearson y predicciones con la prueba de análisis de regresión lineal.

\section{RESULTADOS}

Como se puede apreciar en la Tabla 1 las dimensiones del involucramiento laboral alcanzan puntajes más altos en Compromiso y Fluir en el trabajo, así como en Involucramiento como medida global, siendo en general, valores moderados los que se alcanzan en las demás dimensiones, aunque los valores más bajos se registraron en el Factor negativo y Preocupación. 
Tabla 1 .

Estadísticos descriptivos

\begin{tabular}{|c|c|c|c|c|c|c|c|c|c|c|c|c|c|}
\hline & 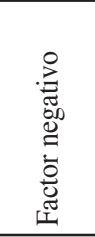 & 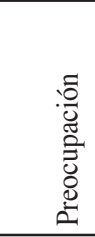 & 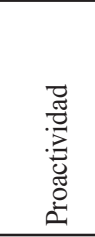 & 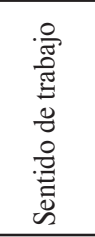 & 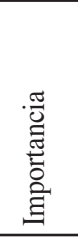 & 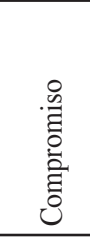 & 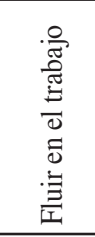 & 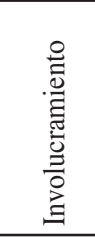 & 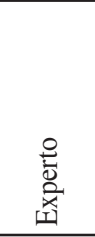 & 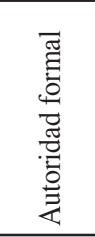 & $\begin{array}{l}\frac{0}{\frac{\pi}{0}} \\
\frac{0}{\Sigma}\end{array}$ & 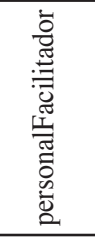 & 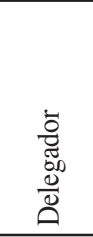 \\
\hline Media & 12.15 & 9.70 & 10.35 & 11.65 & 5.47 & 6.98 & 11.73 & 68.05 & 43.42 & 42.44 & 44.01 & 43.67 & 40.37 \\
\hline Mediana & 12 & 10 & 11 & 12 & 5 & 7 & 12 & 68 & 43.5 & 43 & 45 & 45 & 41 \\
\hline Moda & 14 & 11 & 11 & 11 & 5 & 6 & 11 & 66 & 43 & 42 & 46 & 45 & 41 \\
\hline Desv. típ. & 2.38 & 1.71 & 2.12 & 1.97 & 1.28 & 1.80 & 2.08 & 7.51 & 5.70 & 5.41 & 6.11 & 6.62 & 6.31 \\
\hline Varianza & 5.69 & 2.93 & 4.51 & 3.90 & 1.66 & 3.26 & 4.33 & 56.49 & 32.49 & 29.35 & 37.42 & 43.86 & 39.81 \\
\hline Asimetría & -0.24 & -0.22 & -0.32 & -0.78 & 0.17 & -0.08 & -1.52 & -0.42 & -1.20 & -1.03 & -1.30 & -1.09 & -0.43 \\
\hline Curtosis & 0.82 & -0.01 & -0.35 & 1.79 & 0.37 & -0.65 & 4.38 & 1.19 & 4.29 & 2.88 & 4.18 & 2.63 & -0.09 \\
\hline Mínimo & 4 & 5 & 5 & 4 & 2 & 2 & 3 & 41 & 16 & 19 & 14 & 15 & 23 \\
\hline Máximo & 20 & 14 & 15 & 17 & 9 & 10 & 15 & 88 & 53 & 53 & 55 & 56 & 56 \\
\hline
\end{tabular}

En lo que respecta a los estilos de enseñanza, se tiene que el estilo que obtiene una media más alta es el Modelo personal, seguido de Facilitador, el estilo Experto, el de Autoridad formal y el Delegador. Esto sugiere que los profesores se preocupan por brindar un modelo profesional coherente a sus estudiantes, actuando como facilitadores en el proceso de transferencia del aprendizaje y tomando como base su experiencia profesional y su dominio de las áreas en las que se desenvuelven. Muy pocos delegan sus funciones a través de actividades docentes, es decir, los profesores asumen su función en el proceso enseñanzaaprendizaje.

\section{Experto $\square$ Autoridad formal $\square$ Modelo personal $\square$ Facilitador $\square$ Delegador}

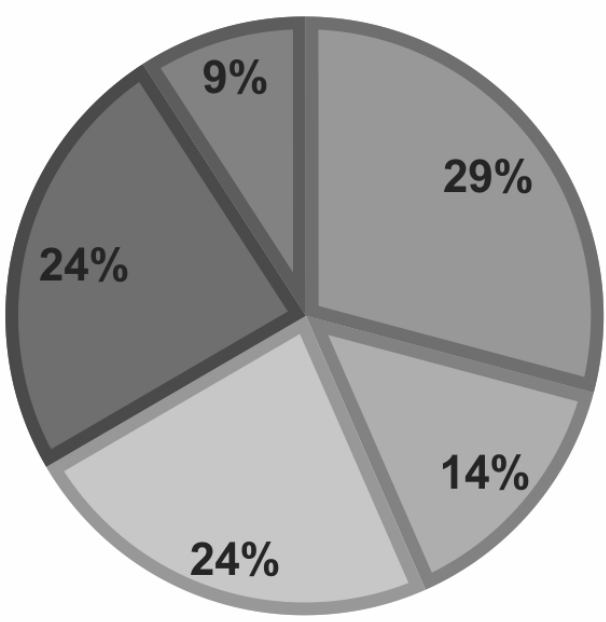

Figura 1. Estilos de enseñanza

Los porcentajes de los Estilos de enseñanza más dominantes entre los profesores universitarios, se muestran en la Gráfica 1, donde se aprecia que el estilo más dominante es el Experto con $29.17 \%$, seguido del Facilitador con $24.17 \%$, el Modelo personal con $23.33 \%$, el Autoridad formal con $14.17 \%$ y el Delegador con $9.17 \%$. 
Tabla 2.

Comparación de medias

\begin{tabular}{|c|c|c|c|c|c|c|c|}
\hline & Sexo & $\mathbf{N}$ & Media & Desviación típ. & $\mathbf{t}$ & gl & $\mathbf{p}$ \\
\hline Factor negativo & $\begin{array}{l}\text { Varón } \\
\text { Mujer }\end{array}$ & $\begin{array}{l}72 \\
41\end{array}$ & $\begin{array}{l}11.888 \\
12.536\end{array}$ & $\begin{array}{l}2.509 \\
2.225\end{array}$ & -1.372 & 111 & 0.172 \\
\hline Preocupación & $\begin{array}{l}\text { Varón } \\
\text { Mujer }\end{array}$ & $\begin{array}{l}72 \\
41\end{array}$ & $\begin{array}{r}10.069 \\
9.097\end{array}$ & $\begin{array}{l}1.630 \\
1.800\end{array}$ & 2.933 & 111 & 0.004 \\
\hline Proactividad & $\begin{array}{l}\text { Varón } \\
\text { Mujer }\end{array}$ & $\begin{array}{l}72 \\
41\end{array}$ & $\begin{array}{r}10.5 \\
9.902\end{array}$ & $\begin{array}{l}2.076 \\
2.188\end{array}$ & 1.442 & 111 & 0.151 \\
\hline Sentido de trabajo & $\begin{array}{l}\text { Varón } \\
\text { Mujer }\end{array}$ & $\begin{array}{l}72 \\
41\end{array}$ & $\begin{array}{l}11.541 \\
11.804\end{array}$ & $\begin{array}{l}1.702 \\
2.522\end{array}$ & -0.660 & 111 & 0.510 \\
\hline Importancia & $\begin{array}{l}\text { Varón } \\
\text { Mujer }\end{array}$ & $\begin{array}{l}72 \\
41\end{array}$ & $\begin{array}{l}5.430 \\
5.536\end{array}$ & $\begin{array}{l}1.172 \\
1.534\end{array}$ & -0.412 & 111 & 0.680 \\
\hline Compromiso & $\begin{array}{l}\text { Varón } \\
\text { Mujer }\end{array}$ & $\begin{array}{l}72 \\
41\end{array}$ & $\begin{array}{l}7.111 \\
7.097\end{array}$ & $\begin{array}{l}1.588 \\
2.047\end{array}$ & 0.039 & 111 & 0.968 \\
\hline Fluir & $\begin{array}{l}\text { Varón } \\
\text { Mujer }\end{array}$ & $\begin{array}{l}72 \\
41\end{array}$ & $\begin{array}{l}11.861 \\
11.463\end{array}$ & $\begin{array}{l}1.878 \\
2.470\end{array}$ & 0.962 & 111 & 0.337 \\
\hline Involucramiento & $\begin{array}{l}\text { Varón } \\
\text { Mujer }\end{array}$ & $\begin{array}{l}72 \\
41\end{array}$ & $\begin{array}{l}68.402 \\
67.439\end{array}$ & $\begin{array}{l}6.737 \\
9.162\end{array}$ & 0.639 & 111 & 0.523 \\
\hline Experto & $\begin{array}{l}\text { Varón } \\
\text { Mujer }\end{array}$ & $\begin{array}{l}72 \\
41\end{array}$ & $\begin{array}{l}44.347 \\
41.731\end{array}$ & $\begin{array}{l}4.463 \\
7.496\end{array}$ & 2.327 & 111 & 0.021 \\
\hline Autoridad formal & $\begin{array}{l}\text { Varón } \\
\text { Mujer }\end{array}$ & $\begin{array}{l}72 \\
41\end{array}$ & $\begin{array}{l}42.930 \\
41.463\end{array}$ & $\begin{array}{l}4.795 \\
6.637\end{array}$ & 1.355 & 111 & 0.177 \\
\hline Modelo personal & $\begin{array}{l}\text { Varón } \\
\text { Mujer }\end{array}$ & $\begin{array}{l}72 \\
41\end{array}$ & $\begin{array}{l}44.416 \\
43.268\end{array}$ & $\begin{array}{l}5.422 \\
7.483\end{array}$ & 0.940 & 111 & 0.349 \\
\hline Facilitador & $\begin{array}{l}\text { Varón } \\
\text { Mujer }\end{array}$ & $\begin{array}{l}72 \\
41\end{array}$ & $\begin{array}{l}43.819 \\
43.536\end{array}$ & $\begin{array}{l}6.215 \\
7.658\end{array}$ & 0.213 & 111 & 0.831 \\
\hline Delegador & $\begin{array}{l}\text { Varón } \\
\text { Mujer }\end{array}$ & $\begin{array}{l}72 \\
41\end{array}$ & $\begin{array}{l}40.611 \\
40.365\end{array}$ & $\begin{array}{l}6.050 \\
7.048\end{array}$ & 0.195 & 111 & 0.845 \\
\hline
\end{tabular}

En la Tabla 2 se tiene que los profesores varones tienen medias más altas en comparación con las mujeres en Preocupación por el trabajo, Compromiso, Fluir en el trabajo, Involucramiento, el estilo de enseñanza Experto, Autoridad formal, Modelo personal, Facilitador y Delegador; mientras que las mujeres tienen medias más altas en Sentido del trabajo, Importancia del trabajo. Sin embargo, solo en Preocupación por el trabajo y en el estilo de enseñanza Experto, estas medidas fueron significativas $(\mathrm{p}<0.05)$, lo que significa que los varones se preocupan más por su trabajo que las mujeres y tienen un estilo de enseñanza basado en su expertise. 
Tabla 3.

Análisis de varianza según grado académico

\begin{tabular}{|c|c|c|c|c|c|c|}
\hline & & Suma de cuadrados & gl & Media cuadrática & $\mathbf{F}$ & Sig. \\
\hline Factor negativo & $\begin{array}{l}\text { Inter-grupos } \\
\text { Intra-grupos } \\
\text { Total }\end{array}$ & $\begin{array}{r}7.683 \\
628.226 \\
635.909\end{array}$ & $\begin{array}{r}2 \\
108 \\
110\end{array}$ & $\begin{array}{l}3.841 \\
5.816\end{array}$ & 0.660 & 0.518 \\
\hline Preocupación & $\begin{array}{l}\text { Inter-grupos } \\
\text { Intra-grupos } \\
\text { Total }\end{array}$ & $\begin{array}{r}3.128 \\
335.214 \\
338.3423\end{array}$ & $\begin{array}{r}2 \\
108 \\
110\end{array}$ & $\begin{array}{l}1.564 \\
3.103\end{array}$ & 0.503 & 0.605 \\
\hline Proactividad & $\begin{array}{l}\text { Inter-grupos } \\
\text { Intra-grupos } \\
\text { Total }\end{array}$ & $\begin{array}{r}4.286 \\
482.488 \\
486.774\end{array}$ & $\begin{array}{r}2 \\
108 \\
110\end{array}$ & $\begin{array}{l}2.143 \\
4.467\end{array}$ & 0.479 & 0.620 \\
\hline Sentido de trabajo & $\begin{array}{l}\text { Inter-grupos } \\
\text { Intra-grupos } \\
\text { Total }\end{array}$ & $\begin{array}{r}1.514 \\
403.476 \\
404.991\end{array}$ & $\begin{array}{r}2 \\
108 \\
110\end{array}$ & $\begin{array}{l}0.757 \\
3.735\end{array}$ & 0.202 & 0.816 \\
\hline Importancia & $\begin{array}{l}\text { Inter-grupos } \\
\text { Intra-grupos } \\
\text { Total }\end{array}$ & $\begin{array}{r}5.169 \\
184.523 \\
189.693\end{array}$ & $\begin{array}{r}2 \\
108 \\
110\end{array}$ & $\begin{array}{l}2.584 \\
1.708\end{array}$ & 1.512 & 0.224 \\
\hline Compromiso & $\begin{array}{l}\text { Inter-grupos } \\
\text { Intra-grupos } \\
\text { Total }\end{array}$ & $\begin{array}{r}4.734 \\
337.535 \\
342.270\end{array}$ & $\begin{array}{r}2 \\
108 \\
110\end{array}$ & $\begin{array}{l}2.367 \\
3.125\end{array}$ & 0.757 & 0.471 \\
\hline Fluir & $\begin{array}{l}\text { Inter-grupos } \\
\text { Intra-grupos } \\
\text { Total }\end{array}$ & $\begin{array}{r}5.204 \\
484.389 \\
489.585\end{array}$ & $\begin{array}{r}2 \\
108 \\
110\end{array}$ & $\begin{array}{l}2.602 \\
4.485\end{array}$ & 0.580 & 0.561 \\
\hline Involucramiento & $\begin{array}{l}\text { Inter-grupos } \\
\text { Intra-grupos } \\
\text { Total }\end{array}$ & $\begin{array}{r}65.571 \\
5982.392 \\
6047.963\end{array}$ & $\begin{array}{r}2 \\
108 \\
110\end{array}$ & $\begin{array}{l}32.785 \\
55.392\end{array}$ & 0.591 & 0.555 \\
\hline Experto & $\begin{array}{l}\text { Inter-grupos } \\
\text { Intra-grupos } \\
\text { Total }\end{array}$ & $\begin{array}{r}109.496 \\
3556.071 \\
3665.567\end{array}$ & $\begin{array}{r}2 \\
108 \\
110\end{array}$ & $\begin{array}{l}54.748 \\
32.926\end{array}$ & 1.662 & 0.194 \\
\hline Autoridad formal & $\begin{array}{l}\text { Inter-grupos } \\
\text { Intra-grupos } \\
\text { Total }\end{array}$ & $\begin{array}{r}92.309 \\
3257.059 \\
3349.369\end{array}$ & $\begin{array}{r}2 \\
108 \\
110\end{array}$ & $\begin{array}{l}46.154 \\
30.157\end{array}$ & 1.530 & 0.221 \\
\hline Modelo personal & $\begin{array}{l}\text { Inter-grupos } \\
\text { Intra-grupos } \\
\text { Total }\end{array}$ & $\begin{array}{r}17.091 \\
4271.142 \\
4288.234\end{array}$ & $\begin{array}{r}2 \\
108 \\
110\end{array}$ & $\begin{array}{r}8.545 \\
39.547\end{array}$ & 0.216 & 0.806 \\
\hline Facilitador & $\begin{array}{l}\text { Inter-grupos } \\
\text { Intra-grupos } \\
\text { Total }\end{array}$ & $\begin{array}{r}31.722 \\
4940.511 \\
4972.234\end{array}$ & $\begin{array}{r}2 \\
108 \\
110\end{array}$ & $\begin{array}{l}15.861 \\
45.745\end{array}$ & 0.346 & 0.707 \\
\hline Delegador & $\begin{array}{l}\text { Inter-grupos } \\
\text { Intra-grupos } \\
\text { Total }\end{array}$ & $\begin{array}{r}94.666 \\
4484.702 \\
4579.369\end{array}$ & $\begin{array}{r}2 \\
108 \\
110\end{array}$ & $\begin{array}{l}47.333 \\
41.525\end{array}$ & 1.139 & 0.323 \\
\hline
\end{tabular}

También se realizaron comparaciones en función del grado académico de los profesores, y se encontró que no había diferencias significativas en base a este criterio, para lo cual se aplicó el análisis de varianza (Tabla 3).
Sin embargo, al hacer las comparaciones a través del análisis de varianza, en función de la profesión de los profesores universitarios, se encontró que existen diferencias significativas en el Factor negativo, Preocupación por el trabajo, el estilo de enseñanza 
de Modelo personal y el estilo Facilitador, a favor de los profesores que son Educadores de profesión. Esto sugiere que estos profesionales se preocupan más por hacer mejor su trabajo docente, y tratan de ser modelos para sus alumnos, facilitando su aprendizaje (Tabla $4)$.

Tabla 4.

Análisis de varianza en función de la profesión

\begin{tabular}{|c|c|c|c|c|c|c|}
\hline & & Suma de cuadrados & gl & Media cuadrática & $\mathbf{F}$ & Sig. \\
\hline Factor negativo & $\begin{array}{l}\text { Inter-grupos } \\
\text { Intra-grupos } \\
\text { Total }\end{array}$ & $\begin{array}{r}87.251 \\
548.658 \\
635.909\end{array}$ & $\begin{array}{r}8 \\
102 \\
110\end{array}$ & $\begin{array}{r}10.906 \\
5.379\end{array}$ & 2.027 & 0.050 \\
\hline Preocupación & $\begin{array}{l}\text { Inter-grupos } \\
\text { Intra-grupos } \\
\text { Total }\end{array}$ & $\begin{array}{r}50.828 \\
287.513 \\
338.342\end{array}$ & $\begin{array}{r}8 \\
102 \\
110\end{array}$ & $\begin{array}{l}6.353 \\
2.818\end{array}$ & 2.254 & 0.029 \\
\hline Proactividad & $\begin{array}{l}\text { Inter-grupos } \\
\text { Intra-grupos } \\
\text { Total }\end{array}$ & $\begin{array}{r}53.278 \\
433.496 \\
486.774\end{array}$ & $\begin{array}{r}8 \\
102 \\
110\end{array}$ & $\begin{array}{l}6.659 \\
4.249\end{array}$ & 1.567 & 0.143 \\
\hline Sentido de trabajo & $\begin{array}{l}\text { Inter-grupos } \\
\text { Intra-grupos } \\
\text { Total }\end{array}$ & $\begin{array}{r}18.715 \\
386.275 \\
404.990\end{array}$ & $\begin{array}{r}8 \\
102 \\
110\end{array}$ & $\begin{array}{l}2.339 \\
3.787\end{array}$ & 0.617 & 0.761 \\
\hline Importancia & $\begin{array}{l}\text { Inter-grupos } \\
\text { Intra-grupos } \\
\text { Total }\end{array}$ & $\begin{array}{r}8.603 \\
181.089 \\
189.693\end{array}$ & $\begin{array}{r}8 \\
102 \\
110\end{array}$ & $\begin{array}{l}1.075 \\
1.775\end{array}$ & 0.605 & 0.771 \\
\hline Compromiso & $\begin{array}{l}\text { Inter-grupos } \\
\text { Intra-grupos } \\
\text { Total }\end{array}$ & $\begin{array}{r}34.839 \\
307.431 \\
342.270\end{array}$ & $\begin{array}{r}8 \\
102 \\
110\end{array}$ & $\begin{array}{l}4.354 \\
3.014\end{array}$ & 1.444 & 0.187 \\
\hline Fluir & $\begin{array}{l}\text { Inter-grupos } \\
\text { Intra-grupos } \\
\text { Total }\end{array}$ & $\begin{array}{r}43.889 \\
445.696 \\
489.585\end{array}$ & $\begin{array}{r}8 \\
102 \\
110\end{array}$ & $\begin{array}{l}5.486 \\
4.369\end{array}$ & 1.255 & 0.275 \\
\hline Involucramiento & $\begin{array}{l}\text { Inter-grupos } \\
\text { Intra-grupos } \\
\text { Total }\end{array}$ & $\begin{array}{r}656.000 \\
5391.963 \\
6047.963\end{array}$ & $\begin{array}{r}8 \\
102 \\
110\end{array}$ & $\begin{array}{l}82.000 \\
52.862\end{array}$ & 1.551 & 0.149 \\
\hline Experto & $\begin{array}{l}\text { Inter-grupos } \\
\text { Intra-grupos } \\
\text { Total }\end{array}$ & $\begin{array}{r}396.346 \\
3269.221 \\
3665.567\end{array}$ & $\begin{array}{r}8 \\
102 \\
110\end{array}$ & $\begin{array}{l}49.543 \\
32.051\end{array}$ & 1.545 & 0.150 \\
\hline Autoridad formal & $\begin{array}{l}\text { Inter-grupos } \\
\text { Intra-grupos } \\
\text { Total }\end{array}$ & $\begin{array}{r}456.217 \\
2893.151 \\
3349.369\end{array}$ & $\begin{array}{r}8 \\
102 \\
110\end{array}$ & $\begin{array}{l}57.027 \\
28.364\end{array}$ & 2.010 & 0.052 \\
\hline Modelo personal & $\begin{array}{l}\text { Inter-grupos } \\
\text { Intra-grupos } \\
\text { Total }\end{array}$ & $\begin{array}{r}803.355 \\
3484.878 \\
4288.234\end{array}$ & $\begin{array}{r}8 \\
102 \\
110\end{array}$ & $\begin{array}{r}100.419 \\
34.165\end{array}$ & 2.939 & 0.005 \\
\hline Facilitador & $\begin{array}{l}\text { Inter-grupos } \\
\text { Intra-grupos } \\
\text { Total }\end{array}$ & $\begin{array}{r}793.724 \\
4178.509 \\
4972.234\end{array}$ & $\begin{array}{r}8 \\
102 \\
110\end{array}$ & $\begin{array}{l}99.215 \\
40.965\end{array}$ & 2.421 & 0.019 \\
\hline Delegador & $\begin{array}{l}\text { Inter-grupos } \\
\text { Intra-grupos } \\
\text { Total }\end{array}$ & $\begin{array}{r}609.010 \\
3970.358 \\
4579.369\end{array}$ & $\begin{array}{r}8 \\
102 \\
110\end{array}$ & $\begin{array}{l}76.126 \\
38.925\end{array}$ & 1.955 & 0.059 \\
\hline
\end{tabular}


El procesamiento de la información también implicó un análisis correlacional de las variables cuantitativas de estudio, lo cual supuso la aplicación de la prueba de Pearson. En la Tabla 5 se pueden ver las correlaciones. Vemos que el Factor negativo del Involucramiento se relaciona con el Sentido del trabajo y el Involucramiento de manera positiva y moderada. La dimensión de Preocupación por el trabajo también se relaciona con el Involucramiento de manera moderada, mientras que la Proactividad se relaciona moderada y positivamente con el Sentido de trabajo y el Fluir en el trabajo, y más fuertemente con el Involucramiento. El Sentido del trabajo se relaciona de manera moderada y positiva con el Fluir en el trabajo y de manera fuerte con el Involucramiento.

Tabla 5.

Correlaciones de estilos de enseñanza e involucramiento laboral

\begin{tabular}{|c|c|c|c|c|c|c|c|c|c|c|c|c|c|c|}
\hline & 宽 & 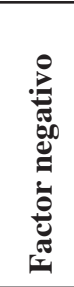 & 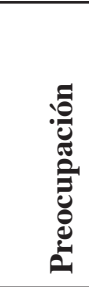 & 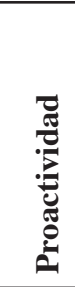 & 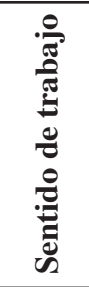 & 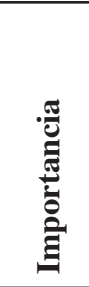 & 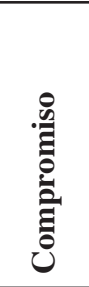 & 竎 & 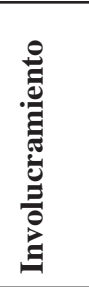 & 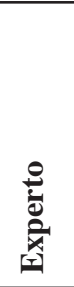 & 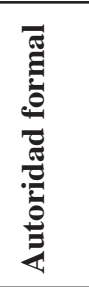 & 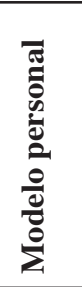 & 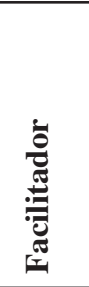 & 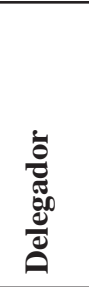 \\
\hline 1 & 1 & 0.08 & -0.00 & 0.00 & -0.01 & -0.15 & 0.00 & -0.02 & -0.01 & 0.06 & 0.07 & 0.04 & 0.08 & 0.10 \\
\hline 2 & & 1 & 0.06 & 0.21 & 0.31 & 0.07 & 0.25 & 0.09 & 0.57 & 0.11 & 0.13 & 0.10 & 0.12 & 0.15 \\
\hline 3 & & & 1 & 0.15 & 0.05 & -0.03 & 0.19 & 0.11 & 0.37 & 0.05 & -0.06 & -0.03 & -0.01 & -0.07 \\
\hline 4 & & & & 1 & 0.35 & 0.07 & 0.17 & 0.32 & 0.62 & 0.19 & 0.17 & 0.20 & -0.02 & 0.07 \\
\hline 5 & & & & & 1 & 0.14 & 0.24 & 0.55 & 0.71 & 0.20 & 0.10 & 0.15 & 0.07 & 0.10 \\
\hline 6 & & & & & & 1 & -0.04 & 0.15 & 0.28 & 0.16 & 0.15 & 0.16 & 0.06 & 0.16 \\
\hline 7 & & & & & & & 1 & 0.29 & 0.55 & 0.19 & 0.01 & 0.10 & 0.17 & 0.17 \\
\hline 8 & & & & & & & & 1 & 0.67 & 0.19 & 0.14 & 0.17 & 0.06 & 0.05 \\
\hline 9 & & & & & & & & & 1 & 0.28 & 0.17 & 0.22 & 0.11 & 0.16 \\
\hline 10 & & & & & & & & & & 1 & 0.60 & 0.65 & 0.49 & 0.45 \\
\hline 11 & & & & & & & & & & & 1 & 0.72 & 0.57 & 0.54 \\
\hline 12 & & & & & & & & & & & & 1 & 0.73 & 0.65 \\
\hline 13 & & & & & & & & & & & & & 1 & 0.71 \\
\hline 14 & & & & & & & & & & & & & & 1 \\
\hline
\end{tabular}

La Importancia en el trabajo se relaciona directa y moderadamente con el Involucramiento, al igual que el Compromiso en el trabajo se relaciona con el Fluir en el trabajo. Sin embargo, se relaciona de manera moderada alta con el Involucramiento, del mismo modo que la dimensión de Fluir en el trabajo. El involucramiento se relaciona con el estilo de enseñanza Experto de manera baja y positiva, mientras que todos los estilos de enseñanza se relacionan entre sí de manera positiva y moderada, siendo las correlaciones más altas, en el caso del estilo Experto con el estilo de Modelo personal, el estilo Autoridad formal con el estilo Modelo personal, el estilo Modelo personal con el estilo Facilitador, y el estilo Facilitador con el estilo Delegador. Por otro lado, el estilo de enseñanza Modelo personal se relacionó débilmente pero significativamente con la Proactividad y el Involucramiento. 
Tabla 6.

Análisis de regresión

\begin{tabular}{|c|c|c|c|c|c|}
\hline & \multicolumn{2}{|c|}{ Coeficientes no estandarizados } & \multirow{2}{*}{$\begin{array}{c}\text { Coeficientes estandarizados } \\
\text { Beta }\end{array}$} & \multirow[t]{2}{*}{$\mathrm{t}$} & \multirow[t]{2}{*}{ Sig } \\
\hline & B & Error típ. & & & \\
\hline (Constante) & 51.721 & 5.981 & & 8.647 & 0.000 \\
\hline Experto & 0.326 & 0.160 & 0.247 & 2.034 & 0.044 \\
\hline Autoridad formal & -0.068 & 0.187 & -0.049 & -0.364 & 0.715 \\
\hline Modelo personal & 0.186 & 0.204 & 0.151 & 0.910 & 0.364 \\
\hline Facilitador & -0.175 & 0.168 & -0.154 & -1.042 & 0.299 \\
\hline Delegador & 0.112 & 0.159 & 0.094 & 0.702 & 0.483 \\
\hline
\end{tabular}

$\mathrm{p}<0.045$

VI: Estilos de enseñanza Experto, Modelo Personal, Autoridad formal, Facilitador y Delegador

VD: Involucramiento en el trabajo

Finalmente, se realizó un análisis de regresión lineal para valorar los efectos de unas variables sobre otras, de este modo, primero se practicaron regresiones, considerando a cada uno de los estilos de enseñanza como variables dependientes y las dimensiones del involucramiento como variables independientes, pero en ningún caso, los modelos de regresión fueron significativos, ni las variables independientes tuvieron poder predictivo sobre los estilos de enseñanza. Sin embargo, luego se realizó una regresión lineal considerando a los estilos de enseñanza como variables independientes y el involucramiento como variable dependiente. Dicho modelo resultó significativo $(\mathrm{p}<$ 0.045) y reveló que el estilo de enseñanza Experto tiene efectos positivos y significativos sobre el involucramiento laboral $(\mathrm{p}<0.044)$, lo que quiere decir que los profesores universitarios caracterizados por un estilo de enseñanza Experto son los que más se involucran con su trabajo docente (ver Tabla 6).

\section{DISCUSIÓN}

En el presente estudio se planteó como objetivo, el análisis descriptivo, comparativo, correlacional y predictivo de los estilos de enseñanza y el involucramiento en el trabajo de los profesores de una universidad privada, ubicada en la ciudad de Arequipa.

En primer lugar, podemos apreciar que los profesores de la universidad presentan niveles moderados de involucramiento laboral, con niveles altos de Compromiso y Fluir en el trabajo. En el primer caso, esto supone que disfrutan de su trabajo y se sienten "absorbidos" por las actividades que realizan en su jornada laboral, siendo esta una condición esencial para el bienestar psicológico del trabajador (Csikszentmihalyi, 2003). En el segundo caso, el compromiso con el trabajo es un componente esencial del involucramiento, que implica el grado en que el trabajador asume las metas organizacionales (Khan, Jam, Akbar, Khan \& Hijazi, 2011; Ekmekci, 2011). Tanto el compromiso como el fluir en el trabajo, fueron los factores más elevados del grupo de profesores universitarios, lo que podría indicar que sus condiciones laborales y organizacionales de la universidad donde laboran son óptimas, o que tienen una marcada disposición hacia el trabajo, mediada por factores personales.

En ese sentido, se encontró que los puntajes en la dimensión de Preocupación fueron significativamente mayores entre las profesoras mujeres, ello posiblemente se deba a la sobre carga que tiene la mujer al desempeñar tanto roles laborales y familiares, que no tienen tanto peso en el varón (Uzonwanne, 2014). Aunque también podrían indicar una tendencia hacia la preocupación, pues algunas investigaciones señalan que las mujeres tienen puntajes más altos en ansiedad y trastornos afectivos en comparación con los varones (Zegarra, \& Cazorla, 2012). Asimismo, no se hallaron diferencias entre los factores del involucramiento laboral en 
función del grado académico, pero sí de la profesión, siendo los profesores universitarios que tienen el título profesional de docente, quienes se preocupan más por su trabajo.

Se puede decir que los profesores de esta universidad se sienten involucrados con su trabajo, y quizá esto de deba a la cultura organizacional de esta casa de estudios, así como al apoyo recibido, pues en otros estudios se ha visto que tanto la cultura organizacional (Rizvi, 2013) como el apoyo social (Mishra \& Shyam, 2005) y el liderazgo de los jefes (Nazem \& Mozaiini, 2014) tienen un impacto significativo en el involucramiento laboral.

Con respecto a los estilos de enseñanza del profesor, se tomó como modelo teórico la propuesta de Anthony Grasha, que distingue cinco estilos de enseñanza: el experto, el de autoridad formal, el modelo personal, el facilitador y el delegador (Grasha, 2000). Los resultados indican que el estilo de enseñanza más dominante fue el de Experto, seguido del Facilitador y el Modelo personal, es decir, los estilos del cluster tres de Grasha (1995). Esto sugiere que los profesores universitarios dominan los temas que enseñan, brindan acompañamiento pertinente a sus estudiantes y se preocupan por darles un buen ejemplo.

A nivel comparativo, se encontraron diferencias significativas en el estilo de enseñanza Experto, con medias mayores para los varones, lo que supone que los profesores de sexo masculino tienen mayor dominio de los temas que enseñan. En otros estudios, también se reportan diferencias en cuanto al sexo de los profesores, pero estas se focalizan más en el estilo de Modelo personal y Facilitador (Grasha, 1990). En cuanto a la profesión de los profesores, se encontró que quienes son profesores de carrera tienen medias más altas en los estilos Modelo personal y Facilitador, lo cual puede estar relacionado con la formación que reciben durante su preparación para ser profesores, pues se les inculcan modelos constructivistas que valoran más estas dimensiones de los estilos de enseñanza (Borgobello, Peralta \& Roselli, 2010).

En ese sentido, un factor muy influyente en el desempeño docente, está dado por las habilidades sociales del profesor (Reyes, 2016), que son base de los estilos de enseñanza Modelo Personal y Facilitador, sin embargo, enfoques muy focalizados en estos aspectos, no siempre son bien valorados por los estudiantes (Kassaian, \& Ayatollahi, 2010). Para el caso de nuestros datos, el estilo de enseñanza Experto podría ser un contrapeso de estas características, que brindan un modelo docente más eficiente, aunque no se haya evaluado la productividad del profesor y su rendimiento, a través del aprovechamiento académico de los alumnos.

A nivel correlacional, se encontró que la Proactividad se relaciona con el Sentido de trabajo, el Fluir y el Involucramiento, mientras que el Sentido del trabajo y el Compromiso se relacionan con el fluir en el trabajo y el Involucramiento. Estas dimensiones son determinantes en el involucramiento, ya que tienen impacto en la motivación del trabajador (Saeidi \& Iravani, 2016), la satisfacción laboral (Gilkar \& Darzi, 2012) y en el civismo organizacional (Saxena \& Saxena, 2015). De ahí su importancia en el ámbito laboral (Lawler \& Hall, 1970).

Los estilos de enseñanza se relacionaron entre sí de manera fuerte y moderada, siendo las relaciones más resaltantes las del estilo Experto con el estilo de Modelo personal, el estilo Autoridad formal con el estilo Modelo personal, el estilo Modelo personal con el estilo Facilitador, y el estilo Facilitador con el estilo Delegador. Las relaciones entre el cruce de variables reportaron que el estilo de enseñanza experto se relaciona con el involucramiento laboral de manera fuerte y significativa, lo que también se corroboró con el análisis de regresión, pues de todos los estilos de enseñanza, el que mejor predice el involucramiento laboral es el estilo Experto, con una valencia positiva y siendo el modelo significativo ( $\mathrm{p}<0.045)$. En cambio, cuando se consideraron los estilos de enseñanza como variables dependientes y las dimensiones del involucramiento como variables independientes, no hubo modelos significativos, ni se registró un impacto estadísticamente significativo del involucramiento en los estilos de enseñanza.

Esto último es un indicador de que los estilos de enseñanza sí pueden tener un impacto en el involucramiento del trabajador, quizá porque entran a tallar ciertos aspectos de la personalidad (Grasha, 
1995; Khurshid \& Aurangzeb, 2012). De hecho, el involucramiento en el trabajo también se ha relacionado con ciertos factores de la personalidad, como la estabilidad emocional, la responsabilidad y la cordialidad (Liao \& Lee, 2009). En ese sentido, si bien el presente estudio, aporta al conocimiento de la realidad de los profesores universitarios, que es muy necesario investigar (Arias, 2013), no ha valorado otras variables como la personalidad, la motivación, y otras de naturaleza similar que sería importante conocer.

Sin embargo, las variables escogidas han sido poco estudiadas en el medio local y nacional, pues si bien hay estudios sobre los estilos de aprendizaje de los estudiantes universitarios de Arequipa (Portilla, \& Barreda, 2002; Arias, 2011, 2014; Arias, Zegarra, y Justo, 2014), solo existe un estudio sobre los estilos de enseñanza (Pastor, 2017). En ese sentido, nuestro trabajo hace un aporte teórico relevante, tanto para el campo de la educación, como del trabajo, dentro del marco de la enseñanza superior, que en los últimos años se ha visto duramente confrontada por los ranking nacionales e internacionales, donde las universidades peruanas ocupan puestos muy bajos (Piscoya, 2006).

En ese sentido, es responsabilidad de los profesores universitarios, el fomentar una mejor cultura académica y para ello, el conocimiento de sus estilos de enseñanza y su dedicación al alumnado (Grasha, 1985) así como valorar las concepciones que tienen del aprendizaje son esenciales (Sánchez, 2005). Igualmente importante, es conocer su grado de involucramiento laboral (Huselid \& Day, 1991), ya que se ha visto que el involucramiento laboral se forma desde el compromiso que se tiene en el ámbito educativo (Sancerni, Peiró, González-Roma, \& Meliá, 1989); por ello, un estilo de enseñanza asociado el involucramiento, podría fomentar un mayor compromiso de parte de los estudiantes, que a futuro, se reflejaría en su desempeño laboral.

Entre las principales limitaciones que podemos señalar en la siguiente investigación, se tiene la imposibilidad de generalizar los resultados en otras universidades de Arequipa o del Perú, debido al tamaño de la muestra; y las desventajas propias de la técnica empleada en la recolección de datos, pues la aplicación de pruebas psicométricas, puede verse influida por sesgos de deseabilidad social, el efecto halo, falta de veracidad, etc. Consideramos, sin embargo, que dada la novedad de las variables estudiadas, la presente investigación sienta un precedente importante, en el estudio de los estilos de enseñanza y el involucramiento laboral de los profesores universitarios; en tal sentido, es necesario replicar la presente investigación en diversos contextos universitarios y con nuevas variables de estudio.

\section{REFERENCIAS}

Aguilera, E. (2014). La percepción de la comunicación en el estudio de los estilos de enseñanza universitarios. Journal of Learning Styles, 7(14), 2-19.

Aguilera,E.,\&Ortiz,E.(2011).Elnivelpsicopedagógico en docentes universitarios, su repercusión en los perfiles de estilos de aprendizaje. Revista Estilos de Aprendizaje, 7(7), 1-11.

Andújar, C. A. y Martínez-Lugo, M.E. (1996). El nivel en que se involucran los empleados en el trabajo. Análisis psicométrico de la Escala de Lodahl y Kejner mediante las teorías clásica y moderna de la medición. Revista Latinoamericana de Psicología, 28(1), 109-134.

Arias, W. L. (2011). Estilos de aprendizaje en estudiantes universitarios y sus particularidades en función de la carrera, el género y el ciclo de estudios. Revista Estilos de Aprendizaje, 8(2), 112-135.

Arias, W. L. (2013). Crisis de la universidad en el Perú: Un problema de su naturaleza y su identidad. Educación, 19(1), 23-39.

Arias, W.L.(2014).Estilos de aprendizaje e inteligencia en estudiantes universitarios de Arequipa, Perú. Journal of Learning Styles, 7(14), 88-107.

Arias, W. L., \& Jiménez, N. A. (2013). Síndrome de burnout en profesores de educación básica regular de Arequipa. Educación, 22(42), 53-76.

Arias, W. L. \&, Jiménez, N. A. (2017). Análisis psicométrico de la Escala de Involucramiento en el Trabajo de Lodahl y Kejner en trabajadores de Arequipa. Industrial Data. (Artículo sometido a revisión). 
Arias, W. L., Zegarra, J., \& Justo, O. (2014). Estilos de aprendizaje y metacognición en estudiantes universitarios de psicología de Arequipa. Liberabit, 20(2), 267-279.

Ato, M., López, J. J., \& Benavente, A. (2013). Un sistema de clasificación de los diseños de investigación en psicología. Anales de Psicología, 29(3), 1038-1059.

Biswas, S. (2009). Psychological climate as a predictor of job involvement. Management Insight, 5(1), 74-79.

Blau, G. J., \& Boal, K. B. (1987). Conceptualizing how job involvement and organizational commitment affects turnover and absenteeism. The Academy of Management Review, 12, 288-300.

Borgobello,A., Peralta, N., \& Roselli, N. (2010). Estilo docente universitario en relación al tipo de clase y la disciplina enseñada. Liberabit, 16(1), 7-16.

Castellano, E., Cifré, E., Spontón, C., Medrano, L. A., Maffei,L. (2013). Emociones positivas y negativas en la predicción del burnout y engagement en el trabajo. Revista Peruana de Psicología y Trabajo Social, 2(1), 75-88.

Ching-Sheue, F. U. (2015). The effect of emotional labor on job involvement in preschool teachers: Verifying the mediating effect of psychological capital. The Turkish Online Journal of Education Technology, 14(3), 145-156.

Chi-Shun, L., \& Cheng-Wen, L. (2009). An empirical study of employee job involvement and personality traits: The case of Taiwan. International Journal of Economics and Management, 3(1), 22-36.

Coloma, C. R., Manrique, L., Revilla, D. M., \& Tafur, R. (2008). Estudio descriptivo de los estilos de aprendizaje de docentes universitarios. Revista Estilos de Aprendizaje, 1(1), 124-142.

Csikszentmihalyi, M. (2003). Fluir en los negocios. Barcelona: Kairós.

Denazis, J. M., \& Óscar, G. (2000). La indagación en los procesos de enseñanza en la Facultad de Ingeniería de la Universidad de Buenos Aires. Psicothema, 12(2), 182-186.

Deosthalee, P. G. (2004). Relationship of work involvement with size of company, nature of appointment, and length of service employees.
Pakistan Journal of Psychological Research, 19(1-2), 15-23.

Ekmekçi, A. K. (2011). A study on involvement and commitment of employees in Turkey. Journal of Public Administration and Policy Research, 3(3), 68-73.

Gilkar, N. A., \& Darzi, J. A. (2012). Employee job involvement and sense of participation influence on job satisfaction: an empirical evidence. International Journal of economics and Management Resecarch, 2(8), 1-8.

Grasha, A. F. (1985). The dynamics of one-on-one teaching. College Teaching, 50(4), 139-146.

Grasha, A. F. (1990). The naturalistic approach to learning style. College Teaching, 55(3), 106-109.

Grasha, A. F. (1995). Teaching with style: The integration of teaching and learning styles in the classroom. Essays on Teaching Excellence, 7(5), 1-6.

Grasha, A. (1996). Teaching with style: A practical guide to enhancing learning by understanding teaching and learning styles. Pittsburgh, USA: Alliance Publishers.

Grasha, A. F. (2000). A matter of style: The teacher as Expert Formal Authority, Personal Model, Facilitator and Delegator. College Teaching, 42(4), 142-149.

Hernández, R., Fernández, C., \& Baptista, P. (2011). Metodología de la investigación. México: McGraw-Hill.

Hui-Ling, Y., Yu-Hsiu, K., \& Yi-Ching, H. (2006). The job self-efficacy and job involvement of clinical nursing teachers. Journal of Nursing Research, 14(3), 237-248

Huselid, M. A., \& Day, N. (1991). Organizational commitment, job involvement, and turnover: A substantive and methodological analysis. Journal of Applied Psychology, 76, 380-391.

Kassaian, Z., \& Ayatollahi, M. A. (2010). Teaching styles and optimal guidance in English language major. Quarterly Journal of Research and Planning in Higher Education, 55, 131-152.

Khan, T. I., Jam, F. A., Akbar, A., Khan, M. B., \& Hijazi, S. T. (2011). Job involvement as predictor 
of employee commitment: Evidence from Pakistan. International Journal of Business and Management, 6(4), 252-262.

Khurshid, F. \& Aurangzeb, W. (2012). Teaching styles $\&$ adolescents' psychosocial development. Global Journal of Human Social Science, 12(10), 19-28.

Lawler, E, \& Hall, D. (1970). Relationship of job characteristics to job involvement, satisfaction, and intrinsic motivation. Journal of Applied Psychology, 54, 305-312.

Liao, C. S., \& Lee, C. W. (2009). An empirical study of employee job involvement and personality traits: The case of Taiwan. International Journal of Economics and Management, 3(1), 22-36.

Lodahl, T. M., \& Kejner, M. (1965). The definition and measurement of job involvement. Journal Applied Psychology, 49, 24-33.

López-Araujo, B., Osca, A., \& Peiró, J. M. (2007). El papel modulador de la implicación con el trabajo en la relación entre el estrés y la satisfacción laboral. Psicothema, 19(1), 81-87.

Lucia-Casademunt, A. M.; Morales-Gutiérrez, A. C. y Ariza-Montes, J. A. (2012). La implicación emocional en el puesto de trabajo: Un estudio empírico. Intangible Capital, 8(2), 364-405.

Mahajan, K. (2015). An empirical study of job involvement and personality traits. International Journal of Scientific Research, 4(1), 56-58.

Martínez, P. (2009). Estilos de enseñanza: Conceptualización e investigación (en función de los estilos de aprendizaje de Alonso, Gallego y Honey). Revista Estilos de Aprendizaje, 3(3), 319.

Martínez-Lugo, M. E. (1988). La involucración con el trabajo y su relación con la edad, el locus de control y los años de servicio. Revista Latinoamericana de Psicología, 20(2), 137-148.

Martínez-Lugo, M. E. (1990). Variables asociadas a la involucración en el trabajo. Revista Latinoamericana de Psicología, 22(2), 211-222.

Meneses, P. E. (2013). Relación entre estilos de aprendizaje y estilos de pensamiento de profesores universitarios. Revista de Estilos de Aprendizaje, 11(11), 80-99.
Mishra, P. C., \& Shyam, M. (2005). Social support and job involvement in prison officers. Journal of the Indian Academy of Applied Psychology, 31(1-2), 7-11.

Nazem, F., \& Mozaiini, M. (2014). Investigating the relationship between leadership style (transformational and pragmatic) and employee's job involvement at Islamic Azad University of Roudehen, Damavand, Pardis, Boomehen and Firuzkuh to provide an appropriate model. Bulletin of Environmental, Pharmacology and Life Sciences, 3(4), 82-88.

Nwibere,B.M.(2014).Interactive relationship between job involvement, job satisfaction, organizational citizenship behavior, and organizational commitment in Nigerian universities. International Journal of Management and Sustainability, 3(6), 321-340.

Pastor, I. (2017). Relación entre estilos de enseñanza $\mathrm{y}$ tipos de personalidad en docentes de nivel superior. Perspectiva Educacional, 56(1), 62-83.

Piscoya, L. (2006). Ranking Universitario en el Perú. Lima: ANR.

Polanco-Bueno, R. (1994). Observación y retroalimentación del comportamiento docente en el salón de clases: sus efectos sobre el desempeño de profesores universitarios. Revista Latinoamericana de Psicología, 26(1), 35-49.

Polanco-Bueno, R. (1999). El estilo de aprendizaje como predictor del desempeño docente en profesores de áreas administrativo-sociales y de ingeniería. Revista Latinoamericana de Psicología, 31(3), 527-536.

Portilla,Ch.,\& Barreda,A.(2002).Estilos de aprendizaje en estudiantes universitarios de las escuelas de artes e ingenierías de la Universidad Nacional de San Agustín. Revista Institucional, 1, 75-88.

Reyes, M. E. (2016). Relación entre habilidades sociales y desempeño docente desde la percepción de los estudiantes adultos de universidad privada de Lima, Perú. Revista Digital de Investigación en Docencia Universitaria, 10(2). doi: 10.19083/ ridu. 10.465

Rizvi, F. M. (2013). Job involvement as related to organizational culture and social support among 
nurses of private and government hospitals. Interdisciplinary Journal of Contemporary Research in Business, 5(7), 564-574.

Rojas, L. I., Zárate, J. F., \& Lozano, A. (2016). La relación entre los estilos de aprendizaje de los estudiantes y los estilos de enseñanza del profesor en un grupo de alumnos de primer semestre del nivel universitario. Journal of Learning Styles, 9(17), 174-205.

Saeidi, K., \& Iravani, M. R. (2016). Predicting the job involvement based on job motivation variable among experts and supervisors of Isfahan Cement Company. The Caspian Sea Journal, 10(1), 278282.

Sancerni, M. D., Peiró, J. M., González-Roma, V. \& Meliá, J. L. (1989). El compromiso con el trabajo en la transición de la escuela al mundo laboral. Revista de Psicología Social, 4(3), 247-259.

Sánchez, L. (2005). Concepciones de aprendizaje de profesores universitarios y profesionales no docentes: Un estudio comparativo. Anales de Psicología, 21(2), 231-243.

Saxena, S., \& Saxena, R. (2015). Impact of job involvement and organizational commitment on organizational citizenship behavior. International
Journal of Management, Business and Research, 5(1), 19-30.

Shaari, A. S., Ysoff, N. M., Ghazali, I. M., Osman, R. H., \& Dzahir, N. F. M. (2014). The relationship between lecturer's teaching style and student's academic engagement. Procedia Social and Behavioral Sciences, 118, 10-20.

Segura, F. M. (2011). Tendencias en los estilos de aprendizaje de estudiantes y profesores en instituciones educativas venezolanas de bachillerato y formación técnica superior. Revista Estilos de Aprendizaje, 7(7), 12-35.

Uzonwanne, F. C. (2014). Marital stress and job involvement among pilots and other employees at an Air Force Base in Lagos, Nigeria. European Journal of Humanities and Social Sciences, 31(1), 1-22.

Van Wyk, R., Boshoff; A. B., \& Cilliers, F. (2003). The prediction of job involvement for pharmacists and accountants. SA Journal of Industrial Psychology, 29(3), 61-67.

Zegarra, J., \& Cazorla, E. (2012). Estudio clínicodescriptivo en el Centro de Salud Mental Moisés Heresi, en Arequipa. Revista de Psicología, 2, 1325.

Fecha de recepción: 6 de febrero, 2018

Fecha de aceptación: 10 de mayo, 2018 